\title{
Leiomyosarcoma of the inferior vena cava with renal metastasis: An unusual case and diagnostic challenge
}

\author{
Garson Chan, MD; ${ }^{*}$ Tadeusz Kroczak, MD; ${ }^{\dagger}$ Darrel Drachenberg, MD, FRCSC ${ }^{\dagger}$ \\ *Division of Urology, St. Joseph's Health Care, Western University, London, ON; †Section of Urology, Department of Surgery, St. Boniface Hospital, University of Manitoba, Winnipeg, MB
}

Cite as: Can Urol Assoc J 2014;8(9-10):358-60. http://dx.doi.org/10.5489/cuaj.2119 Published online October 13, 2014.

\section{Abstract}

Leiomyosarcomas are exceedingly rare malignant tumours with a very poor prognosis. We present a 78-year-old patient with a small renal and paracaval mass. Surgery included right open partial nephrectomy and resection of the paracaval mass to treat the presumed renal cell carcinoma. Pathology revealed the tumour to be leiomyosarcoma (LMS). This is only the second documented report of an inferior vena cava LMS presenting along with an ipsilateral renal mass.

\section{Case report}

A 78-year-old male with a history of intermediate-risk prostate cancer treated with neoadjuvant and adjuvant androgen deprivation therapy, as well as external beam radiotherapy 1 year ago, presented at a follow-up appointment with significant right-sided flank and abdominal pain. Physical examination revealed tenderness in the right flank without detectable peripheral lymphadenopathy. Routine blood work was normal and prostate serum antigen (PSA) was $0.91 \mu \mathrm{g} / \mathrm{L}$. Computed tomography (CT) and magnetic resonance imaging revealed an enhancing exophytic lesion off the inferior pole of the right kidney measuring $2.1 \mathrm{~cm}$ in diameter (Fig. 1a), as well as a 1.8-cm mass anterior to inferior vena cava (IVC) (Fig. 1b) suspicious for an enlarged lymph node. The right renal vein did not appear to be involved. These lesions were not likely metastatic prostate cancer since PSA was $0.91 \mu \mathrm{g} / \mathrm{L}$.

Consultation with urology was initiated for further management of the renal lesion. A positron emission tomography scan was ordered to assess the activity of the caval lymph node. It revealed a hypermetabolic IVC lymph node, as well as several bilateral hypermetabolic hilar lung abnormalities.
A bone scan revealed no skeletal metastasis. Repeat CT scan showed growth of the IVC node to $2.2 \mathrm{~cm}$ and bilateral pulmonary nodules. The suspicious renal mass remained stable at $1.8 \mathrm{~cm}$.

The patient agreed to an open partial nephrectomy and retroperitoneal lymph node dissection as the anterior caval mass was not amendable to biopsy. During the preoperative period, another CT scan showed the IVC node had increased in size to $2.7 \mathrm{~cm}$, while the renal mass remained stable. The renal mass was removed from the right kidney. Intraoperatively, the anterior caval mass involved the IVC and about $30 \%$ of the circumference was resected. Pathology revealed a high grade $2.3-\mathrm{cm}$ renal mass and a 3.8-cm caval leiomyosarcoma (LMS) (Fig. 2a). Diagnosis was confirmed with positive smooth muscle cell markers for actin, desmin and vimentin (Fig. 2b). Based on pathology, the final diagnosis was primary IVC LMS with metastasis to the ipsilateral kidney.

Postoperatively the patient was seen by the medical oncology team; the patient declined any adjuvant therapy. The patient developed multifocal pulmonary metastases 3 years after his surgery and succumbed to his disease a year later.

\section{Discussion}

Leiomyosarcomas are extremely rare and often aggressive malignant tumours that are associated with a dismal prognosis. ${ }^{1-5}$ Management is not well-established due to rare presentations and limited literature..$^{6,7}$ The presentation is often subtle, ranging from abdominal pain, palpable masses, constitutional symptoms or even Budd-Chiari syndrome. ${ }^{8}$ In our case, the lesions were considered malignant based on imaging and were initially thought to represent RCC with ipsilateral solitary lymph node metastasis.

Due to the aggressive nature and size of the IVC lesion, the LMS was considered of caval origin rather than a renal primary. LMS of renal origin is very rare, with even fewer case reports than IVC LMS. ${ }^{9}$ Furthermore, renal LMS and 


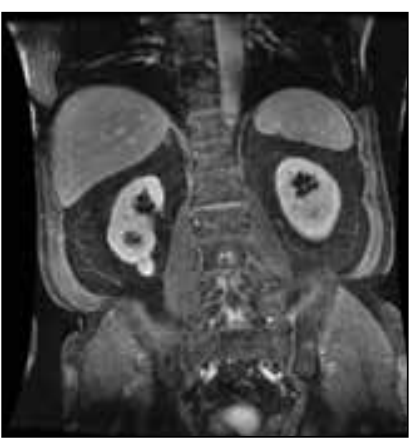

Fig. 1a. Coronal magnetic resonance imaging post-gadolinium fat suppressed T1 weighted image showing a lower pole right renal mass.

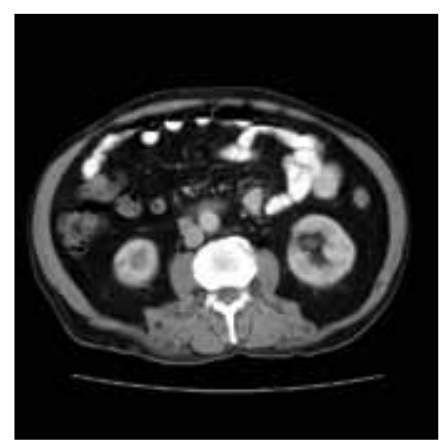

Fig. 1b. Axial infused computed tomography scan with a mass anterior to inferior vena cava.

RCC are notoriously difficult to differentiate, which made our case such a diagnostic challenge. Given that the anterior IVC lesion showed interval growth preoperatively, while the renal lesion was stable and the IVC lesion was much larger than the renal lesion, we confirmed a diagnosis of IVC LMS with metastasis to the ipsilateral kidney. In addition, the literature suggests that LMS should be classified as IVC in origin if the involved IVC segment must also be resected with the malignant tumour. ${ }^{10} \mathrm{LMS}$ of the IVC usually metastasizes to the adjacent kidney by local invasion through the renal vessels; however, in this case the renal vessels were not involved. The current treatment of such tumours is radical en bloc resection. ${ }^{11,12}$ To our knowledge, this is only the second documented case in the urological literature of IVC LMS with metastasis to the ipsilateral kidney presenting as primary RCC. ${ }^{13}$

Our patient did not receive adjuvant chemotherapy or radiation. In cases of LMS of the IVC, neoadjuvant therapy may improve resectability. In this case, the primary was

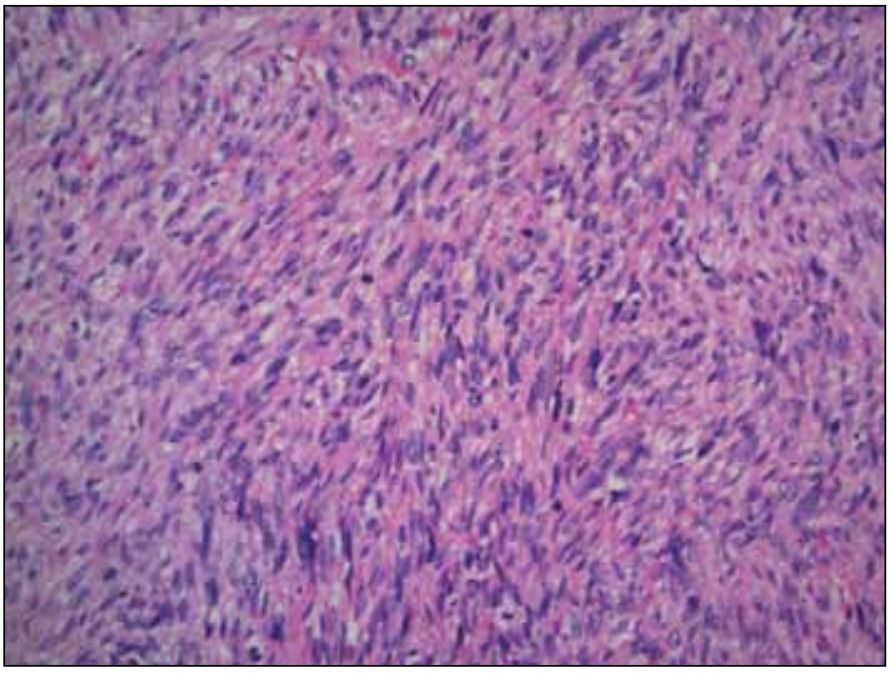

Fig. 2a. Caval mass stained with hematoxylin and eosin (20x magnification). Spindle cells arranged in intersecting fascicles are seen along with multiple mitotic figures reflecting the proliferative nature of the tumour. renal so neoadjuvant therapy was not considered. Adjuvant therapy with radiation has improved median survival when compared to observation and even greater benefit when combined with chemotherapy and compared to radiation alone. ${ }^{14}$ Chemotherapy using adriamycin-ifosfamide or combination with radiation therapy is also an option with survival benefit. ${ }^{15}$

\section{Conclusion}

Caval LMS is very rare and may present in a variety of ways. It is very difficult to distinguish it from other renal masses until the pathologic analysis. Radical resection followed by adjuvant treatment should be considered the optimal therapeutic strategy for tumours without metastasis at the initial diagnosis.

Competing interests: Dr. Chan, Dr. Kroczak and Dr. Drachenberg declare no competing financial or personal interests.

This paper has been peer-reviewed.

\section{References}

1. Dhawan S, Chopra P, Dhawa S. Primary renal leiomyosarcoma: A diagnostic challenge. Urol Ann 2012;4:48-50.

2. Destito A, Candidi MO, et al. Two atypical cases of renal leiomyosarcoma: Clinical picture, diagnosis and therapy. Arch Ital Urol Androl 1998;70:199-201.

3. Mingoli A, Cavallaro A, Sapienza P, et al. International registry of inferior vena cava leiomyosarcoma: Analysis of a world series on 218 patients. Anticancer Res 1996;16(5B):3201-5.

4. Hollenbeck ST, Grobmyer SR, Kent KC, et al. Surgical treatment and outcomes of patients with primary inferior vena cava leiomyosarcoma. J Am Coll Surg 2003;197:575-9. http://dx.doi.org/10.1016/ S1072-7515(03)00433-2

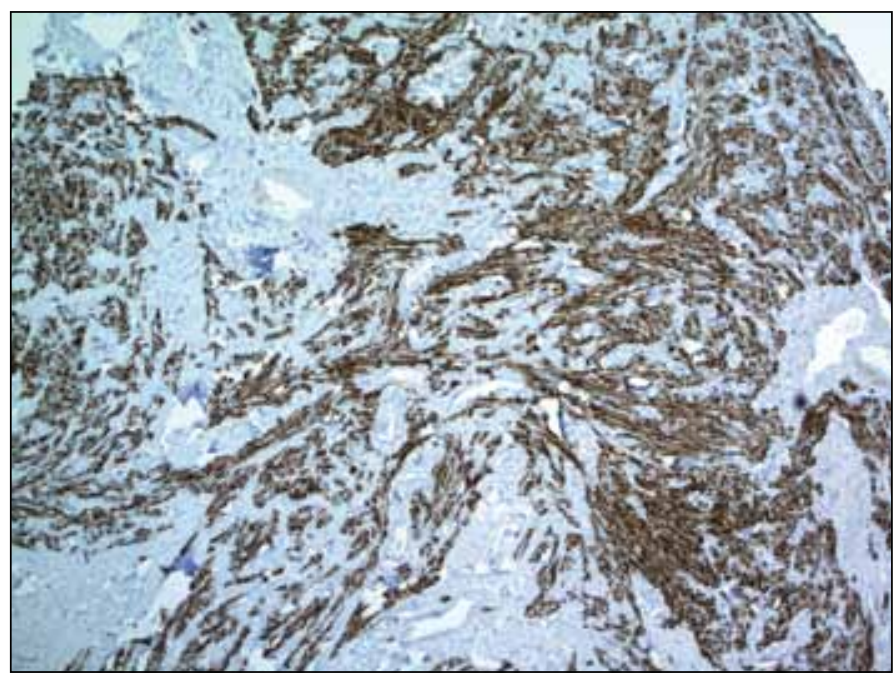

Fig. 2b. Renal mass staining positive for desmin, confirming myogenic origin (4x magnification). 
Chan et al.

5. Cocuzza M, Arap S, Lucon AM, et al. Renal leiomyosarcoma treated with partial nephrectomy. Clinics (Sao Paulo) 2005;60:345-6. http://dx.doi.org/10.1590/S1807-59322005000400013

6. Shvarts 0 , Han KR, Lam JS, et al. Primary leiomyosarcoma of the inferior vena cava presenting as a renal mass. Rev Urol 2004;6:39-42.

7. Hines $0 \mathrm{~J}$, Nelson $\mathrm{S}$, Quinones-Baldrich WJ, et al. Leiomyosarcoma of the inferior vena cava: prognosis and comparison with leiomyosarcoma of other anatomic sites. Cancer 1999;85:1077-83. http://dx.doi. org/10.1002/(SICl) 1097-0142(19990301)85:5<1077::AID-CNCR10>3.0.C0;2-0
8. Kieffer $E$, Alaoui $M$, Piefte JC, et al. Leiomyosarcoma of the inferior vena cava: Experience in 22 cases. Ann Surg 2006;244:289-95. http://dx.doi.org/10.1097/01.sla.0000229964.71743.db

Correspondence: Dr. Tadeusz Kroczak, Section of Urology, Department of Surgery, St. Boniface, Hospital University of Manitoba, Winnipeg, MB; umkrocza@cc.umanitoba.ca 